\title{
Commonness of Polycystic Ovarian Syndrome amongst Nominated Cluster of Sudanese Housewives with Fertility Problems
}

\author{
Mohammed A. Gafoor A. Gadir, Mohammed Omer Mohammed Hussein, Mosab Nouraldein \\ Mohammed Hamad*
}

Banoon Center of Obstetrics and Gynecology and Reproduction, Sudan

*Corresponding Author

Mosab Nouraldein Mohammed Hamad

\section{Article History}

Received: 25.11.2020

Accepted: 07.12.2020

Published: 13.12.2020

\begin{abstract}
Polycystic ovary syndrome (PCOS), also known as the Stein-Leventhal syndrome, is one of the most prevalent endocrinopathies amid women of reproductive age. Hyperinsulinemia in combination with pancreatic beta cell dysfunction consequences in an augmented risk of numerous diseases, including type 2 diabetes, hypertension, dyslipidemia, endothelial dysfunction, atherosclerosis and cardiovascular diseases. Insulin also encourages the theca cells of the ovary to make excessive testosterone, which is responsible for the clinical manifestations of hyperandrogenism, 200 Sudanese housewives with fertility problems joined in the study, $39.5 \%$ of them exhibited polycystic ovarian syndrome. Further studies are required with large sample size.
\end{abstract}

Keywords: PCOS, syndrome, reproductive-age, women. Sudan.

\section{INTRODUCTION}

Polycystic ovary syndrome (PCOS), also renowned as the Stein-Leventhal syndrome, is one of the most common endocrinopathies between women of reproductive age. It is estimated that it influences 3-15\% of all women. An abnormality in the ovaries is the primary source of the disorder, but extra agents, such as obesity and environmental factors, affect the progress of individual symptoms [1]. The Rotterdam criterion (2003) are the most extensively used and relevant criteria for the diagnosis of PCOS. The disarray is diagnosed if 2 of the 3 specified conditions are met: (1) .hyperandrogenism (identified by clinical and/or biochemical testing) (2) ovulation abnormalities, and/or (3) 12 or more cysts on one ovary and/or ovarian volume $>10 \mathrm{~mL}$. There are also 2 other descriptions of the syndrome in addition to the Rotterdam criteria. Along with the criteria proposed by the National Institutes of Health [NIH, 2009], a diagnosis of PCOS involves recognition of clinical or biochemical hyperandrogenism and chronic ovulation disorders.

The Androgen Excess Society [2006], conversely, cures hyperandrogenism as the basic PCOS disorder and the prerequisite for a diagnosis, in mixture with one of the 2 remaining Rotterdam criteria [2]. In all these cases, PCOS can be nvestigated after Cushing's syndrome, congenital adrenal hyperplasia and/or androgen-secreting tumors have been ruled out.Based on the Rotterdam criterion, 4 phenotypes of PCOS can be differentiated: 1 classic: hyperandrogenism $(\mathrm{H})$, ovulation disorders $(\mathrm{O})$ and a polycystic ovary $[\mathrm{P}]$ noticed by USG [HOP]; 2 with hyperandrogenism and ovulation disorders, but with a normal ovarian USG image [HO]; 3 with hyperandrogenism and a polycystic ovary USG image, but without ovulation disorders [HP]; 4 with ovulation disorders and a polycystic ovary USG image, but without proof of hyperandrogenism [1-3] .

In spite of these seemingly obvious criteria, the etiology of PCOS remains unidentified, and accurate treatment procedures have not been recognized. PCOS therefore persists to be the object of research and scientific inquiry.

Copyright (C) 2020 The Author(s): This is an open-access article distributed under the terms of the Creative Commons Attribution 4.0 International License (CC BY-NC 4.0) which permits unrestricted use, distribution, and reproduction in any medium for non-commercial use provided the original author and source are credited. 
An imperfection of the ovarian cells (most likely theca cells) is the fundamental reason of PCOS, resulting in extreme androgen synthesis and the clinical and biochemical symptoms of the illness [1, 2]. In the previous study, reference is made to the contribution of genetic factors, counting ethnicity; there is a highe. Occurrence of PCOS in Spanish, Native American and Mexican women [4].

In their unique explanation of the syndrome, Stein and Leventhal highlighted that a high ratio of luteinizing hormone ( $\mathrm{LH})$ to follicle-stimulating hormone $(\mathrm{FSH})$ is one of the basic disorders. It has also been suggested that the original causes of PCOS include increased frequency of gonadotropin-releasing hormone $(\mathrm{GnRH})$ pulses that stimulate the theca cells to produce androgen; reduced levels of FSH (and thus a defect in the late luteal and early follicular phases); insulin resistance via a post-receptor imperfection in the fat tissue and skeletal muscles (abnormal phosphorylation of tyrosine kinase); pancreatic beta-cell dysfunction; and obesity [1, 3].

It is frequently unfeasible to determine definitively what a cause is and what an effect in the progress of PCOS is. Also, it is generally documented that obesity increases menstrual disorders and hyperandrogenism, while weight lessening reduces the clinical signs. Decreased insulin sensitivity is a significant issue in both obese and underweight women with PCOS; it is probable that $50-70 \%$ of women with the condition show insulin resistance of changeable intensities [3].

The effect of genetic factors was emphasized by Davies et al., who confirmed that mothers of women with PCOS are more expected to have a cardiovascular disease and that their risk of hypertension is twice as high as mothers of women without PCOS, while fathers of women with PCOS are twice as expected to have heart disease and 4 times more probable to have experienced cerebral stroke [5]. Tan et al. highlighted the raised likelihood of insulin resistance (IR) connected with certain genes (such as INSIG2 and MC4R) and the particular impact of TCF7L2 SNP on the progress of diabetes mellitus type 2 (DM2) and body weight gain in patients with PCOS (a per-allele weight gain of 1.56 $\mathrm{kg} / \mathrm{m}^{2}$ ) [6]. The etiology of IR was also conversed by Fica et al. who, as emphasizing the complex mechanisms of PCOS, recognized insulin receptor autophosphorylation, reduced levels of phosphatidylinositol-3-kinase in muscle tissue and visceral adiposity as probable methods [7].

Hyperinsulinemia in mixture with pancreatic beta cell dysfunction consequences in an increased risk of numerous illnesses, counting type 2 diabetes, hypertension, dyslipidemia, endothelial dysfunction, atherosclerosis and cardiovascular diseases. Insulin also stimulates the theca cells of the ovary to create excessive testosterone, which is accountable for the clinical symptoms of hyperandrogenism (acne, hirsutism, alopecia) [8].

\section{Methodology}

Study design

Descriptive cross-sectional study

\section{Study period}

January-December 2018

\section{Study population}

Sudanese housewives with fertility disorders, attended to banoon fertility center.

\section{Sample size}

200 participants

\section{Data Collection}

Data collected through well-formed questionnaire.

\section{Ethical approval}

Each participant was informed about the objectives of the study, and they consented to be involved in it.

\section{RESULT}

$69(39.5 \%)$ from the total participants (200), showed polycystic ovarian syndrome.

\section{CONCLUSION AND RECOMMENDATIONS}

We conclude that PCOS represent major problem for many women, which prevent them from their dream to have kids and become mothers. Further studies are needed with large sample size and within long duration of time. 


\section{REFERENCES}

1. Wołczyński, S., \& Zgliczyński, W. (2012). Abnormalities of the menstrual cycle. Large Interna-Endocrinology. 2nd edition. Medical Tribune Poland, Warsaw, 561-567.

2. ESHRE, T. R., \& ASRM-Sponsored PCOS Consensus Workshop Group. (2004). Revised 2003 consensus on diagnostic criteria and long-term health risks related to polycystic ovary syndrome. Fertility and sterility, 81(1), 1925.

3. Milewicz, A. (2013). Reimbursement of metformin for polycystic ovary syndrome. Endokrynologia Polska, 64(5), 409-414.

4. Morotti, E., Battaglia, B., Fabbri, R., Paradisi, R., Venturoli, S., \& Battaglia, C. (2014). Cigarette smoking and cardiovascular risk in young women with polycystic ovary syndrome. International journal of fertility \& sterility, 7(4), 301.

5. Davies, M. J., Marino, J. L., Willson, K. J., March, W. A., \& Moore, V. M. (2011). Intergenerational associations of chronic disease and polycystic ovary syndrome. PLoS One, 6(10), e25947.

6. Tan, S., Scherag, A., Janssen, O. E., Hahn, S., Lahner, H., Dietz, T., ... \& Illig, T. (2010). Large effects on body mass index and insulin resistance of fat mass and obesity associated gene (FTO) variants in patients with polycystic ovary syndrome (PCOS). BMC medical genetics, 11(1), 12.

7. Fica, S., Albu, A., Constantin, M., \& Dobri, G. A. (2008). Insulin resistance and fertility in polycystic ovary syndrome. Journal of medicine and life, 1(4), 415.

8. Traub, M. L. (2011). Assessing and treating insulin resistance in women with polycystic ovarian syndrome. World Journal of Diabetes, 2(3), 33. 